\title{
Finiteness results for flat surfaces: large cusps and short geodesics
}

\author{
John Smillie and Barak Weiss
}

\begin{abstract}
For fixed $g$ and $T$ we show the finiteness of the set of affine equivalence classes of flat surfaces of genus $g$ whose Veech group contains a cusp of hyperbolic co-area less than $T$. We obtain new restrictions on Veech groups: any non-elementary Fuchsian group can appear only finitely many times in a fixed stratum; any non-elementary Veech group is of finite index in its normalizer; and the quotient of $\mathbb{H}$ by a non-lattice Veech group admits arbitrarily large embedded disks. A key ingredient of the proof is the finiteness of the set of affine equivalence classes of flat surfaces of genus $g$ whose Veech group contains a hyperbolic element with eigenvalue less than $T$.
\end{abstract}

Mathematics Subject Classification (2000). 57M50, 37D40.

Keywords. Flat surface, Veech group, Fuchsian group, Markov partition.

\section{Introduction}

Our objects of study are flat surfaces and their affine automorphism groups. These structures arise in the study of rational polygonal billiards, in Thurston's classification of surface diffeomorphisms in connection with measured foliations, and in complex analysis. The class of flat surfaces is subdivided into translation surfaces and halftranslation surfaces, which correspond in the complex analysis literature to abelian and quadratic holomorphic differentials respectively.

Let $\operatorname{Aff}(M)$ denote the affine automorphism group of a flat surface $M$. Let $G=$ $\operatorname{SL}(2, \mathbb{R})$ if $M$ is a translation surface, and $\operatorname{PSL}(2, \mathbb{R})$ if $M$ is a half-translation surface. Taking the differential of an automorphism yields a homomorphism $D: \operatorname{Aff}(M) \rightarrow$ $G$ with finite kernel, whose image $\Gamma_{M}$ is discrete and is called the Veech group of $M$. Alternatively, $\Gamma_{M}$ is the stabilizer, under the $G$-action, of $M$ in the stratum $\mathscr{H}$ of flat surfaces containing $M$. For a typical flat surface $\operatorname{Aff}(M)$ (and hence $\Gamma_{M}$ ) is trivial; however surfaces with non-trivial Veech groups are quite interesting. Much of the interest in the subject was generated by Veech's discovery [Ve2] of Veech groups which are non-arithmetic lattices (recall that $\Gamma$ is called a lattice if $\mathbb{H} / \Gamma$ has finite area). Many additional Veech groups have been described, including infinitely generated ones [HuSc2], [Mc1], and there are many intriguing questions regarding 
groups which may arise as Veech groups. See [HuSc3] for a recent survey of the field, and [SmWe1] for a problem list.

A fundamental question in this regard is to understand the commensurability classes of Fuchsian groups which contain Veech groups (recall that a Fuchsian group is a discrete subgroup of $G$ and two such groups are commensurable if their intersection is of finite index in both). We will describe some restrictions on groups commensurable to a Veech group $\Gamma$ and on the geometry of the corresponding $\mathbb{H} / \Gamma$. The question of which groups within a given commensurability class actually arise as Veech groups is also of considerable interest, see [HuSc1].

It is clear that if $\Gamma$ is a lattice then there is an upper bound on the radius of a maximal embedded disk. We have the following converse, which provides a new characterization of lattice surfaces.

Theorem 1.1. If $\Gamma$ is commensurable to a Veech group and is not a lattice then for every $R \geq 0$, the quotient $\mathbb{H} / \Gamma$ contains an embedded disk of radius $R$.

We derive a purely group-theoretic property of Veech groups:

Theorem 1.2. A non-elementary Fuchsian group commensurable to a Veech group is of finite index in its normalizer.

These theorems follow from some finiteness results which involve an upper bound on either the covolume of a cusp for $\Gamma_{M}$, or the eigenvalue of a hyperbolic element in $\Gamma_{M}$. For a Fuchsian group $\Gamma$, a cusp in $\mathbb{H} / \Gamma$ is determined by a conjugacy class of a maximal parabolic subgroup $P \subset \Gamma$. Associated with this data is the cusp area, i.e., the hyperbolic area of a maximal continuous embedded family of parallel closed horocycles on $\mathbb{H} / \Gamma$. We denote the cusp area by $t_{0}(\Gamma, P)$. Also, when $\Gamma$ is the Veech group of a flat surface $M$, the foliation on $M$ in the direction fixed by $P$ is periodic, i.e. $M$ is decomposed into finitely many maximal embedded metric cylinders. We denote the number of cylinders by $m(M, P)$. Both of these quantities are invariant under affine equivalence. That is, for all $g \in G, \Gamma_{g M}=g \Gamma_{M} g^{-1}$ contains the maximal parabolic subgroup $g P g^{-1}$ and

$$
t_{0}\left(\Gamma_{g M}, g P g^{-1}\right)=t_{0}\left(\Gamma_{M}, P\right), \quad m\left(g M, g P g^{-1}\right)=m(M, P) .
$$

Let

$$
\operatorname{SC}(m, T)=\left\{(M, P): t_{0}\left(\Gamma_{M}, P\right) \leq T, m(M, P)=m\right\},
$$

where $M$ ranges over all flat surfaces, and $P$ ranges over all maximal parabolic subgroups of $\Gamma_{M}$ (SC stands for 'small cusp'). We denote by $\widetilde{\mathrm{SC}}(m, T)$ the corresponding set of affine equivalence classes.

The following holds: 
Theorem 1.3. For any $T>0$ and any $m \in \mathbb{N}$, the set $\widetilde{\mathrm{SC}}(m, T)$ is finite.

Our proof yields an explicit bound on \# $\widetilde{\mathrm{SC}}(m, T)$, see Theorem 4.2.

Let $\mu$ be a Haar measure on $G$ and let $\Gamma$ be a discrete subgroup of $G$. We denote the covolume of $\Gamma$ in $G$ by $\bar{\mu}(\Gamma)$; this number depends only on the conjugacy class of $\Gamma$. We deduce:

Corollary 1.4. Let $\mathscr{H}$ be a stratum (see $\S 2.1$ ) offlat surfaces and let $R, T>0$. Then the following sets are finite.

(i) The set of affine equivalence classes of $M \in \mathscr{H}$ for which $\mathbb{H} / \Gamma_{M}$ contains no embedded ball of radius $R$.

(ii) The set of affine equivalence classes of $M \in \mathscr{H}$ for which $\bar{\mu}\left(\Gamma_{M}\right)<T$.

Note that Veech [Ve2] constructs lattice surfaces $M_{n}$ for all $n \neq 4$ (on different strata) such that $\bar{\mu}\left(\Gamma_{M_{n}}\right)<2 \pi$. Thus one cannot omit the hypothesis that $M$ is contained in a fixed stratum in Corollary 1.4. Assertion (ii) was proved independently by Curt McMullen [Mc2], using the algebraic geometry of moduli space.

Suppose $\Gamma_{M}$ contains a hyperbolic element $h$. We denote the larger eigenvalue of $h$ by $\lambda(h)$ and call it the eigenvalue of $(M, h)$. Also, associated to $h$ are Markov partitions of $M$ (see $\S 5$ ). We let $p=p(M, h)$ be the minimal number of parallelograms in a Markov partition. For $T>0$ and $p \in \mathbb{N}$ we define

$$
\operatorname{SMP}(p, T)=\{(M, h): p(M, h)=p, \lambda(h)<T\}
$$

(SMP stands for 'simple Markov partition'). These quantities are also invariant under affine equivalence: if $h \in \Gamma_{M}$ is hyperbolic then $g h g^{-1} \in \Gamma_{g M}$ is also hyperbolic with $p(M, h)=p\left(g M, g h g^{-1}\right)$ and $\lambda(h)=\lambda\left(g h g^{-1}\right)$. We denote the set of affine equivalence classes in SMP by $\widetilde{\text { SMP. }}$. Repeating a folklore argument ${ }^{1}$ we obtain:

Proposition 1.5 (Thurston, Veech). For a fixed $T>0$ and $p \in \mathbb{N}, \widetilde{\operatorname{SMP}}(p, T)$ is finite.

The existence of Markov partitions is sketched in [FLP] and we explain it in detail in the appendix. In particular we show that bounding $p$ is equivalent to bounding the genus of $M$. Note that by [Pe], there are pairs $\left(M_{n}, h_{n}\right)$ with $\lambda\left(h_{n}\right) \rightarrow 1$, i.e. one cannot omit a bound on $p$ (or on the topology of $M$ ) from the statement.

Recall that the geodesic flow is the restriction of the $G$-action on $\mathscr{H}$ to the oneparameter subgroup of diagonal matrices. There is a bijective correspondence between $G$-orbits of pairs $(M, h)$ as above, and surfaces with a periodic trajectory under

\footnotetext{
${ }^{1}$ The argument is probably due to Thurston. We were unable to find a suitable reference, but see [Th, p. 428] for a hint and [Ry, Theorem 1.7] for more details.
} 
the geodesic flow, with the length of the corresponding trajectory equal to $\log \lambda(h)$. Thus Proposition 1.5 is equivalent to the statement, proved by Veech [Ve1], that the number of periodic geodesic trajectories in $\mathscr{H}$ of length at most $T$ is finite.

For a group $\Gamma$ and $h \in \Gamma$ we write $h^{\Gamma}$ for the conjugacy class of $h$ in $\Gamma$. From Theorem 1.3 and Proposition 1.5 we derive a restriction on Veech groups:

Corollary 1.6. Suppose $\Gamma$ is commensurable to a Veech group. Then for any $T>0$, the following sets are finite:

(I) $\left\{P \subset \Gamma: P\right.$ is a maximal parabolic subgroup, $\left.t_{0}(\Gamma, P) \leq T\right\}$.

(II) $\left\{h^{\Gamma}: h \in \Gamma\right.$ is hyperbolic with $\left.\lambda(h)<T\right\}$.

(III) $\left\{f^{\Gamma}: f \in \Gamma\right.$ is elliptic with cone area at most $\left.T\right\}$.

Here the cone area associated with an elliptic $f \in \Gamma$ is the area of $B\left(z_{f}, R\right) / Z_{\Gamma}(f)$, where $z_{f} \in \mathbb{H}$ is the fixed point of $f, Z_{\Gamma}(f)$ is the centralizer of $f$, and

$$
R=R(f)=\sup \left\{r: B\left(z_{f}, r\right) /\langle f\rangle \rightarrow \mathbb{H} / \Gamma \text { is injective }\right\} .
$$

Given a Fuchsian group, it is natural to ask 'how often' it arises as a Veech group. Cyclic parabolic subgroups are associated with cylinder decompositions and are easily described. In any stratum there are infinitely many of them belonging to different $G$-orbits. Using torus covers one can construct infinitely many flat surfaces $M$, in different strata, with the same non-elementary Veech group - see [He] for infinitely many square-tiled translation surfaces whose Veech group is $\operatorname{SL}(2, \mathbb{Z})$. Also, if $\Gamma=\Gamma_{M}$ and $g \in G$ normalizes $\Gamma$ then also $\Gamma=\Gamma_{g M}$. As an application of our results we show that aside from these simple constructions, each group can only appear finitely many times, namely:

Corollary 1.7. For any stratum $\mathscr{H}$ and any infinite Fuchsian group $\Gamma$ which is not cyclic parabolic, the set

$$
\left\{M \in \mathscr{H}: \Gamma_{M}=\Gamma\right\}
$$

contains finitely many $N$-orbits, where $N$ is the normalizer of $\Gamma$ in $G$. In particular if $\Gamma$ is non-elementary then (2) is finite.

Acknowledgements. We thank Yair Minsky and Yair Glasner for useful discussions. The support of NSF grant DMS-0302357, BSF grant 2004149 and ISF grant 584-04 is gratefully acknowledged. Some of the results of this paper were announced in [SmWe1]. 


\section{Basics}

In this section we set some notation, and collect standard results.

2.1. Flat surfaces. We begin by listing some definitions. For more details we refer the reader to $[\mathrm{MaTa}],[\mathrm{Vo}],[\mathrm{Zo}]$.

Throughout this paper, $S$ denotes a compact connected orientable surface of genus $g$. When $S$ is equipped with the structure of a flat surface or a holomorphic quadratic differential we will usually denote it by $M$. When confusion may arise we will also use $M$ to denote the underlying surface $S$. A flat surface admits several equivalent definitions. It may be thought of as an equivalence class of atlases of charts $\left(U_{\alpha}, \varphi_{\alpha}\right)$ covering all but a finite set $\Sigma=\Sigma_{M} \subset S$ of singularities, such that the transition functions $\varphi_{\alpha} \circ \varphi_{\beta}^{-1}$ are of the form $z \mapsto \pm z+c$, and such that for each $\sigma \in \Sigma$ the charts combine to form $k$-pronged singularity at $\sigma$, where $k=k_{\sigma} \in \mathbb{N}$. If $k_{\sigma}=2$ then $\sigma$ is called a removable singularity or marked point. We always assume that $\Sigma_{M} \neq \varnothing$. Two atlases $M, M^{\prime}$ are compatible if $M \cup M^{\prime}$ is also an atlas satisfying the same conditions. The atlases $M=\left(U_{\alpha}, \varphi_{\alpha}\right), M^{\prime}$ are equivalent if there is a self-homeomorphism $h: S \rightarrow S$ such that $h\left(\Sigma_{M}\right)=\Sigma_{M^{\prime}}$ and $\left(h\left(U_{\alpha}\right), \varphi_{\alpha} \circ h\right)$ is compatible with $M^{\prime}$. A flat surface is called a translation surface, or an abelian differential, if there is a compatible atlas in which all transition functions are of the form $z \mapsto z+c$. A flat surface which is not a translation surface is called a half-translation surface.

If $M$ and $M^{\prime}$ are flat surfaces, an affine equivalence from $M$ to $M^{\prime}$ is a homeomorphism which is affine in each chart. An affine equivalence from $M$ to itself is called an affine automorphism of $M$, and the group of all such maps is denoted by Aff $(M)$. For an affine equivalence $\phi: M \rightarrow M^{\prime}$, the derivative $d \phi_{x}$ at $x \in M$ is well-defined in the case of translation surfaces (i.e. independent of charts around $x$ and $\phi(x)$ ), and in the case of half-translation surfaces, is well-defined up to \pm Id. Additionally it is independent of the point $x$. Clearly $M$ and $M^{\prime}$ are equivalent if and only if there is an affine equivalence between them with derivative Id ( \pm Id) in the case of translation surfaces (half-translation surfaces). Since the area of $M$ is finite, the derivative of an affine automorphism of a flat surface $M$ is contained in $G$, and the group of all derivatives is the Veech group $\Gamma_{M}$.

Fix the data giving the number of singularities, the vector $\vec{k}=\left(k_{\sigma}\right)_{\sigma \in \Sigma}$, and the determination whether or not the flat surfaces are translation surfaces; then the set of equivalence classes of all atlases sharing this data is called a stratum. Each stratum is equipped with a structure of an affine orbifold, which is locally modelled on a relative cohomology group, see [MaSm], [MaTa]. The group $G$ acts on $\mathscr{H}$. In the case of translation surfaces, the action is by post-composition on each chart in an atlas. In the case of half-translation surfaces, for $g \in G$ we may choose a representative in $\operatorname{SL}(2, \mathbb{R})$ and let it act on an atlas by post-composition; the resulting equivalence 
class of half-translation surfaces is well-defined.

A flat surface inherits from the plane a singular foliation on $S$ called the horizontal (resp. vertical) foliation, with each chart foliated into the lines parallel to the $x$-axis (resp. $y$-axis). A saddle connection is a straight segment joining singularities or punctures, with no singularities or punctures in its interior. The set of all saddle connections in direction $\theta$ is denoted by $\mathscr{L}_{M}(\theta)$.

Throughout this paper we identify elements of $G$ with matrices of $\operatorname{SL}(2, \mathbb{R})$. We will need the following four one-parameter subgroups of $G$ :

$$
\begin{array}{rlrl}
g_{t} & =\left(\begin{array}{cc}
e^{t / 2} & 0 \\
0 & e^{-t / 2}
\end{array}\right), & r_{\theta} & =\left(\begin{array}{cc}
\cos \theta & -\sin \theta \\
\sin \theta & \cos \theta
\end{array}\right), \\
h_{s}=\left(\begin{array}{ll}
1 & s \\
0 & 1
\end{array}\right), & \tilde{h}_{s}=\left(\begin{array}{ll}
1 & 0 \\
s & 1
\end{array}\right) .
\end{array}
$$

We say that $\lambda_{1}, \ldots, \lambda_{k} \in \mathbb{R}$ are commensurable if $\lambda_{i} / \lambda_{j} \in \mathbb{Q}$ for all $i, j \in$ $\{1, \ldots, k\}$. If this holds, we denote by $\operatorname{LCM}\left(\lambda_{1}, \ldots, \lambda_{k}\right)$ the smallest positive number which is an integer multiple of all the $\lambda_{i}$.

2.2. Cylinder decompositions, gluing patterns. A cylinder for $M$ is a topological annulus which is isometric to $\mathbb{R} / w \mathbb{Z} \times(0, h)$, for positive $h, w$. It is maximal if it is not contained in a larger cylinder, and this implies that both of its boundary components in $S$ contain singularities. The height, width, and inverse modulus of the cylinder are $h, w$, and $w / h$ respectively, and a curve which wraps around the cylinder parallel to its boundary is called a waist curve. A cylinder decomposition is a decomposition of $M$ into maximal cylinders with disjoint interiors. The waist curves of all the cylinders in a cylinder decomposition are parallel. Two cylinder decompositions of $M$ are called transverse if the slopes of waist curves in each decomposition are different.

2.2.1. Gluing pattern for a pair of cylinder decompositions. Suppose for $i=1,2$ that we have two transverse cylinder decompositions $M=C_{1}^{(i)} \cup \cdots \cup C_{m_{i}}^{(i)}$. For each $i \in\left\{1, \ldots, m_{1}\right\}, j \in\left\{1, \ldots, m_{2}\right\}$, the intersection $C_{i}^{(1)} \cap C_{j}^{(2)}$ consists of $a_{i j}$ parallelograms. The resulting parallelograms have disjoint interiors and boundary identifications. To describe how the parallelograms are attached to each other we follow ideas of [EsOk].

Suppose first that $M$ is a translation surface. In this case one can consistently label the edges of the parallelograms with labels right, left, top, bottom. Label the parallelograms by $1, \ldots, \ell$, let $S_{\ell}$ denote the group of permutations on $\ell$ elements, and define $\sigma_{1}, \sigma_{2} \in S_{\ell}$, where $\sigma_{1}\left(k_{1}\right)=k_{2}$ (resp. $\sigma_{2}\left(k_{1}\right)=k_{2}$ ) if the right (resp. top) edge of the $k_{1}$ th parallelogram is attached to the left (resp. bottom) edge of the $k_{2}$ th parallelogram. 
Replacing the labelling of the parallelograms amounts to replacing $\sigma_{1}, \sigma_{2}$ with $\tau \sigma_{1} \tau^{-1}, \tau \sigma_{2} \tau^{-1}$ for some $\tau \in S_{\ell}$. We denote the equivalence class of $\left(\sigma_{1}, \sigma_{2}\right) \in$ $S_{\ell} \times S_{\ell}$ under simultaneous conjugations by $\left[\left(\sigma_{1}, \sigma_{2}\right)\right]$, and call $\left[\left(\sigma_{1}, \sigma_{2}\right)\right]$ the gluing pattern of $M$ and the given cylinder decompositions. From the gluing pattern it is simple to recover the number of cylinders in each decomposition, the number of connected components in each intersection of cylinders, and the stratum to which $M$ belongs. In particular the following hold:

(i) For $i=1,2, \sigma_{i}$ has $m_{i}$ cycles, and the length of the $j$ th cycle of $\sigma_{1}$ (resp. $\sigma_{2}$ ) is $\sum_{i} a_{i j}$ (resp. $\left.\sum_{i} a_{j i}\right)$.

(ii) The subgroup of $S_{\ell}$ generated by $\sigma_{1}, \sigma_{2}$ acts transitively on $\{1, \ldots, \ell\}$.

Moreover $M$ is completely determined by the dimensions and orientation of the parallelogram and the corresponding gluing pattern; indeed such information gives rise to an explicit atlas of charts as in $\$ 2.1$.

In case $M$ is a half-translation surface, one can define a gluing pattern in a more complicated way by subdividing each rectangle into four 'quarter-tiles'. This will not be used in the current paper.

2.2.2. Gluing pattern for parallelograms. We will also have occasion to consider flat surfaces made up of finitely many parallelograms, glued along edges. We suppose that $M$ is a translation surface which is a union of closed metric parallelograms $P_{1}, \ldots, P_{m}$, with disjoint interiors, such that the two directions of parallel sides are the same for all $P_{i}$ and such that the singularities are on the boundaries of the parallelograms; replacing $M$ with an affinely equivalent surface, let us assume these sides are horizontal and vertical. Let $\xi_{1}, \ldots, \xi_{\ell_{1}}$ (respectively $\eta_{1}, \ldots, \eta_{\ell_{2}}$ ) be a list of the horizontal (resp. vertical) connected components of all intersections $P_{i} \cap P_{j}$, for $i \neq j$, which are not points. That is all the $\xi_{i}$ and $\eta_{j}$ are all segments; if one of them contains a singularity of $M$ in its interior we subdivide it in two, so after finitely many steps the $\xi_{i}$ and $\eta_{j}$ do not contain singularities in their interior. Orienting the $\xi$-edges from left to right and the $\eta$-edges from bottom to top, we obtain a directed graph with two kinds of edges, embedded in $M$ and equipped with two additional structures:

- At each vertex there is a cyclic order for the incident edges, obtained by going around a small neighborhood of the point in $M$ in the counterclockwise direction. Moreover along a cycle, consecutive edges of the same kind have opposite orientations (e.g. if $\eta_{i}$ is incoming and $\eta_{j}$ is the next $\eta$-edge then it is outgoing).

- For each $\xi$ (resp. $\eta$ ) segment there are top and bottom (resp. right and left) labels from $\{1, \ldots, m\}$ indicating the parallelograms glued to the segment on the appropriate side.

We call a graph with these structures a gluing pattern for a decomposition into parallelograms. The gluing pattern obeys certain obvious restrictions. For example, at a 
vertex $v$, if there is no $\xi$ edge between an incoming and an outgoing $\eta$ edge then the 'right' labels of the two $\eta$ edges are the same. Note that the gluing pattern determines the stratum containing $M$. Since each vertex of a gluing pattern is either a corner of a rectangle or a singularity of $M$, there are only finitely many gluing patterns when the number of singularities and the number of parallelograms are fixed.

Also associated with a parallelogram decomposition is the metric data consisting of the lengths of sides of the $P_{i}$ and the lengths of the $\xi$ - and $\eta$-edges. Given the gluing pattern and the lengths of the edges, it is possible to calculate the sidelengths of the $P_{i}$; thus the gluing pattern places some restrictions on the metric information. It is clear that the gluing pattern and the metric data taken together uniquely determine the translation surface $M$.

One could similarly define a gluing pattern for a parallelogram decomposition of a half-translation surface. This will not be used in the present paper and is left to the avid reader.

2.3. Fuchsian groups. A Fuchsian group is a discrete subgroup of $G$. Fixing a Haar measure $\mu$ on $G$, we define the covolume of $\Gamma$ in $G$ as the measure of a measurable fundamental domain for the action of $\Gamma$ on $G$, and denote the covolume by $\bar{\mu}(\Gamma)$. It is easily checked that if $M$ and $M^{\prime}$ are affinely equivalent then $\bar{\mu}\left(\Gamma_{M}\right)=\bar{\mu}\left(\Gamma_{M^{\prime}}\right)$. If $\bar{\mu}\left(\Gamma_{M}\right)<\infty$ then $M$ is called a lattice surface.

An element of a Fuchsian group is called parabolic if it is conjugate to $h_{1}$ or its inverse, and an automorphism $\varphi \in \operatorname{Aff}(M)$ is called parabolic if $D \varphi \in \Gamma_{M}$ is parabolic. The following is well known (see [Ve2, Proposition 2.4]):

Proposition 2.1. If $\varphi \in \operatorname{Aff}(M)$ is parabolic and $D \varphi$ fixes the direction $\theta$, then $M-\mathscr{L}_{M}(\theta)$ is a cylinder decomposition. For any $m$ there is $\kappa=\kappa_{m}$ such that if $m$ is the number of cylinders in the above decomposition, then there is $k \leq \kappa$ such that $\psi=\varphi^{k}$ preserves the cylinders and the corresponding inverse moduli $\mu_{1}, \ldots, \mu_{m}$ for $\psi$ satisfy $\operatorname{LCM}\left(\mu_{1}, \ldots, \mu_{m}\right)=\mu$, where $D \psi=r_{\theta} h_{\mu} r_{-\theta}$ and $n_{i}=\mu / \mu_{i}$ is the number of Dehn twists which $\psi$ induces around a waist curve in the ith cylinder.

For a Fuchsian group $\Gamma$ we now define cusp areas and cone areas. An infinite cyclic subgroup of $G$ generated by a parabolic element is called parabolic. Suppose that $\Gamma$ contains a parabolic subgroup $P$ and is non-elementary, that is, not a finite extension of an abelian group. Suppose also that $P$ is maximal, i.e. not properly contained in a parabolic subgroup of $\Gamma$. Choose an element $g \in G$ such that

$$
g P g^{-1}=\left\langle h_{1}\right\rangle,
$$

and relabelling, replace $P$ and $\Gamma$ by $g P g^{-1}$ and $g \Gamma g^{-1}$ respectively. Let $\mathbb{H}$ be the complex upper half plane, let $\mathbf{i}=\sqrt{-1}$, let $\mathscr{C}_{t}$ denote the image of the disk 
$\left\{z \in \mathbb{H}:\left|z-\frac{t}{2} \mathbf{i}\right|<t / 2\right\}$ in $\mathbb{H} / P$, let $\varphi: \mathbb{H} / P \rightarrow \mathbb{H} / \Gamma$ be the natural map, and let

$$
t_{0}=t_{0}(\Gamma, P)=\sup \left\{t>0: \varphi \mid e_{t} \text { is injective }\right\} .
$$

We will show in Proposition 3.1 that the set in the right-hand side of (4) is nonempty and bounded above, so that $t_{0}$ is well-defined, and that $t_{0}$ does not depend on the choice of $g$ in (3) and satisfies

$$
t_{0}(\Gamma, P)=t_{0}\left(x \Gamma x^{-1}, x P x^{-1}\right)
$$

for $x \in G$. We call $t_{0}$ the cusp area of $P$ in $\Gamma$; a simple computation shows that it is equal to the hyperbolic area of $e_{t_{0}}$.

2.4. Orientation double cover. Suppose $M$ is a half-translation surface. Then there is a topological branched cover $\pi: \widetilde{S} \rightarrow S$ of degree 2 such that the pulled-back flat surface $\widetilde{M}$ is a translation surface called the orientation double cover of $M$, see [DoHu, p. 175]. We have:

Proposition 2.2. Let $\tilde{M} \rightarrow M$ be the orientation double cover of a half-translation surface M. Then:

- Any automorphism of $M$ lifts to an automorphism of $\tilde{M}$, with the same derivative. If a parabolic (resp. hyperbolic) affine automorphism $\varphi$ of $M$ has an associated cylinder decomposition (resp. Markov partition, see §5) with $k$ cylinders (resp. parallelograms) then the lifted automorphism $\tilde{\varphi}$ of $\tilde{M}$ has an associated cylinder decomposition (resp. Markov partition) with at most $2 k$ cylinders (resp. parallelograms).

- Taking orientation double covers is $G$-equivariant, i.e. for $g \in G, g \tilde{M}$ is the orientation double cover of $g M$.

- Given $\tilde{M}$ there are at most finitely many $M$ such that $\tilde{M}$ is the orientation double cover of $M$.

Proof. A proof of the fact that any affine automorphism lifts can be found in $[\mathrm{Ry}$, Theorem 3.2]. The statement about the number of cylinders and rectangles follows immediately from the fact that the degree of the cover is 2 . The second statement is immediate from the construction of the orientation double cover. For the third statement see e.g. [Vo, §5].

2.5. Perron-Frobenius. A matrix $A \in \operatorname{Mat}_{d}(\mathbb{R})$ with all entries non-negative is called non-negative, and a non-negative matrix is called irreducible if for some $k, A^{k}$ has all its entries strictly positive. A vector $\vec{v} \in \mathbb{R}^{d}$ is called positive if all its entries are strictly positive.

We will need the following classical result (see e.g. [Ga]). 
Proposition 2.3. Suppose $A \in \mathrm{Mat}_{d}(\mathbb{R})$ is an irreducible non-negative matrix. Then:

- There is a unique (up to scaling) positive eigenvector $v^{+}$of $A$, and the corresponding eigenspace is one-dimensional.

- Let $\lambda$ be the eigenvalue for which $A v^{+}=\lambda v^{+}$. Then for any other eigenvalue $\beta$ of $A,|\beta|<\lambda$.

We denote the eigenvalue $\lambda$ of Proposition 2.3 by $\lambda(A)$. We will be particularly interested in irreducible non-negative matrices with integer entries.

Proposition 2.4. Given $T>0$ and $d \in \mathbb{N}$, the set

$$
\left\{A \in \operatorname{Mat}_{d}(\mathbb{Z}): A \text { is irreducible non-negative, } \lambda(A)<T\right\}
$$

is finite.

Proof. This can be deduced from [Ga, Vol 1, p. 63], see [PaPe, Proof of Theorem 6].

\section{Some hyperbolic geometry}

This section contains some standard propositions in hyperbolic geometry related to cusp areas and cone areas for which a suitable reference could not be found. We will use the notation introduced in $\$ 2.3$.

Proposition 3.1. Let $\Gamma$ be a Fuchsian group containing the maximal parabolic subgroup $P=\left\langle h_{1}\right\rangle$. Then $X=\left\{t>0: \varphi \mid \mathcal{E}_{t}\right.$ is injective $\}$ is nonempty. If $\Gamma$ is nonelementary then $t_{0}=\sup X<\infty$, and there is $\gamma_{0} \in \Gamma$ which is a conjugate of $h_{1}$, such that for some $s \in \mathbb{R}, h_{s}^{-1} \gamma_{0} h_{s}=\tilde{h}_{-t_{0}^{2}}$. The hyperbolic area of $\varphi_{t}$ is $t_{0}$, and (5) holds for all $x \in G$.

Proof. We denote the right-action of $\Gamma$ on $\mathbb{H}$ by

$$
z \cdot \gamma=\frac{a z+c}{b z+d}, \quad \text { where } \gamma=\left(\begin{array}{ll}
a & b \\
c & d
\end{array}\right) \in \Gamma .
$$

Let $\mathscr{B}_{t}=\left\{z \in \mathbb{H}:\left|z-\frac{t}{2} \mathbf{i}\right|<\frac{t}{2}\right\}$ and let $H_{t}=\partial \mathscr{B}_{t}$. By our conventions $\mathscr{B}_{t}$ projects to $C_{t}$ and $H_{t}$ is the horocycle based at 0 through $t \mathbf{i}$, which projects to a periodic horocycle on $\mathbb{H} / P$. Let $S \subset H_{1}$ be a compact segment whose projection to $\mathbb{H} / P$ contains a full period of the horocycle. Since the action of $\Gamma$ on $\mathbb{H}$ is properly discontinuous, we can write

$$
\left\{\gamma_{1}, \ldots, \gamma_{N}\right\}=\{\gamma \in \Gamma: S \cap S \cdot \gamma \neq \varnothing, 0 \cdot \gamma \neq 0\}
$$


To show that $X \neq \varnothing$, take $0<t \leq 1$ small enough so that $\mathscr{B}_{t}$ does not intersect $\bigcup_{i=1}^{N} \mathscr{B}_{t} \cdot \gamma_{i}$; such $t$ exist because the $\mathscr{B}_{t} \cdot \gamma_{i}$ are not based at 0 . Suppose if possible that for some $\gamma \in \Gamma \backslash P, \mathscr{B}_{t} \cap \mathcal{B}_{t} \cdot \gamma \neq \varnothing$. In particular $\mathscr{B}_{1} \cap \mathscr{B}_{1} \cdot \gamma \neq \varnothing$. It follows from discreteness of $\Gamma$ and maximality of $P$ that $P$ is equal to its own normalizer in $\Gamma$. This implies that $p=0 \cdot \gamma \neq 0$; then $H_{1} \cap H_{1} \cdot \gamma \neq \varnothing$ (see Figure 1) and since any element of $H_{1}$ may be mapped to $S$ by suitable powers of $h_{1}$, there are $j, k \in \mathbb{Z}$ such that $S \cap S \cdot \tilde{\gamma} \neq \varnothing$, where $\tilde{\gamma}=h_{1}^{j} \gamma h_{1}^{k} \in \Gamma$. Thus for some $i \in\{1, \ldots, N\}$ we have $\tilde{\gamma}=\gamma_{i}$ and $\mathscr{B}_{t} \cap \mathscr{B}_{t} h_{1}^{-j} \gamma_{i} h_{1}^{-k} \neq \varnothing$. Since $\mathscr{B}_{t}$ is invariant under $h_{1}$, this contradicts the definition of $t$.

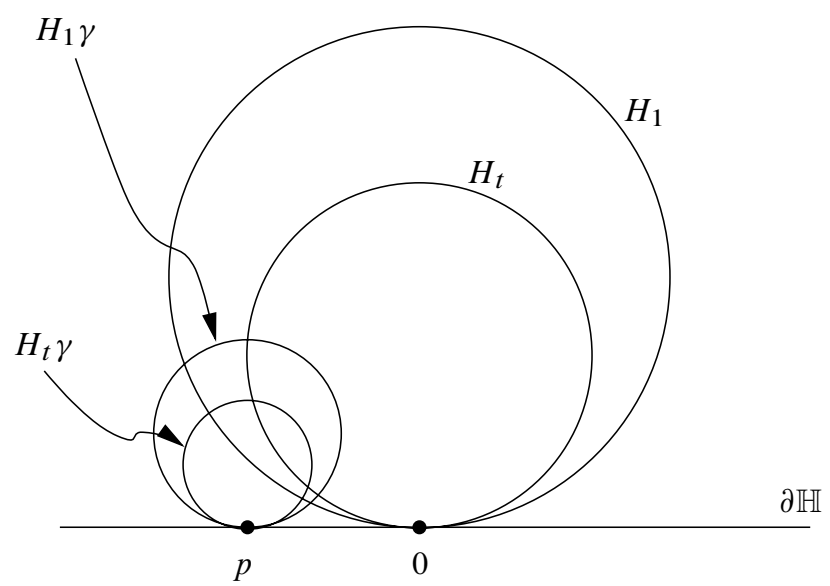

Figure 1. Intersection of horocycles.

Now if $\Gamma$ is nonelementary then it contains $\gamma$ so that $0 \cdot \gamma \neq 0$, so that $H_{1}$ and $H_{1} \cdot \gamma$ are horocycles based at different points. Then for all sufficiently large $t$, $H_{t} \cap H_{t} \cdot \gamma \neq \varnothing$, which shows $\sup X<\infty$. Further, by definition of $t_{0}$, there is $\gamma \in \Gamma$ such that $H_{t_{0}} \cap H_{t_{0}} \cdot \gamma \neq \varnothing$ and $\mathscr{B}_{t_{0}} \cap \mathscr{B}_{t_{0}} \cdot \gamma=\varnothing$, i.e., $H_{t_{0}}$ and $H_{t_{0}} \cdot \gamma$ are horocycles based at different points and tangent to each other. We denote the point of tangency by $p_{0}$.

Since $p_{0} \gamma^{-1} \in H_{t_{0}}$, there is $s_{1}$ such that

$$
p_{0}=p_{0} \cdot x, H_{t_{0}} \cdot x=H_{t_{0}} \cdot \gamma, \quad \text { where } x=h_{s_{1}} \gamma .
$$

We now express $x$ in terms of $t_{0}$, as follows.

Let $s \in \mathbb{R}$ so that $\tilde{p}_{0}=p_{0} \cdot h_{s}=t_{0} \mathbf{i}$. The matrices

$$
a=\left(\begin{array}{cc}
t_{0}^{-1 / 2} & 0 \\
0 & t_{0}^{1 / 2}
\end{array}\right), \quad w=\left(\begin{array}{cc}
0 & -1 \\
1 & 0
\end{array}\right)
$$


satisfy $\tilde{p}_{0} \cdot a=\mathbf{i}=\mathbf{i} \cdot w$, and $H_{1} \cdot w=\tilde{H}$, where $\tilde{H}=\{z \in \mathbb{H}: \operatorname{Im} z=1\}=$ $\left\{\mathbf{i} \cdot \tilde{h}_{s}: s \in \mathbb{R}\right\}$ (see Figure 2). Thus the matrix $x^{\prime}=h_{s} a w a^{-1} h_{-s}$ also satisfies $p_{0}=p_{0} \cdot x^{\prime}, H_{t_{0}} \cdot x^{\prime}=H_{t_{0}} \cdot \gamma$, and this implies

$$
x= \pm h_{s} a w a^{-1} h_{-s} .
$$

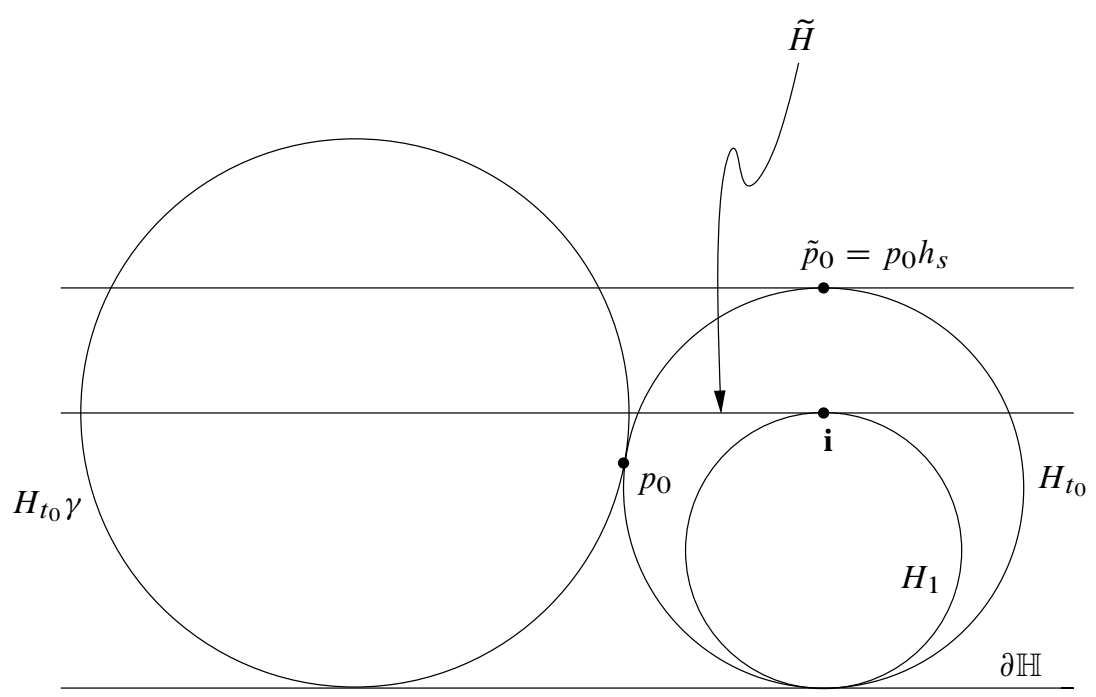

Figure 2. Tangency of $H_{t_{0}}$ and $H_{t_{0}} \gamma$.

Let $\gamma_{0}=x^{-1} h_{1} x=\gamma^{-1} h_{1} \gamma \in \Gamma$. A computation using (7) gives

$$
h_{s}^{-1} \gamma_{0} h_{s}
$$

Finally, note that the hyperbolic area of $\varphi_{t_{0}}$ is the same as that of $\varphi_{t_{0}} \cdot \gamma h_{s}$, which by the above is covered bijectively by $\left\{z=x+\mathbf{i} y \in \mathbb{H}: y \geq t_{0}, 0 \leq x<t_{0}^{2}\right\}$. Hence its area is

$$
\int_{0}^{t_{0}^{2}} \int_{t_{0}}^{\infty} \frac{d y}{y^{2}} d x=t_{0} .
$$

Since $t_{0}(\Gamma, P)$ only depend on the geometry of $\mathbb{H} / \Gamma$, and a conjugation by $x \in G$ affects an isometry on $\mathbb{H} / \Gamma,(5)$ is clear.

Suppose $\Gamma$ is a Fuchsian group containing an elliptic element $f$ such that $\langle f\rangle$ coincides with the centralizer $Z_{\Gamma}(f)$. The corresponding cone in $\mathbb{H} / \Gamma$ is the image of $B\left(z_{f}, R(f)\right)$ under the map $\varphi: \mathbb{H} /\langle f\rangle \rightarrow \mathbb{H} / \Gamma$, where $z_{f} \in \mathbb{H}$ is the fixed point of $f$ and $R(f)$ is defined via (1). 
Proposition 3.2. With the above notation, let $\gamma \in \Gamma$ such that $z_{f} \gamma$ is a closest point to $z_{f}$ in the orbit $z_{f} \Gamma$. Then the commutator $h=[\gamma, f]$ is hyperbolic, its eigenvalue is bounded above by a number depending only on $R(f)$, and the distance from the axis of $h$ to $z_{f}$ is also bounded by a number depending on $R(f)$.

Proof. Write $z=z_{f}, z^{\prime}=z \gamma$ and $f^{\prime}=\gamma f \gamma^{-1}$. Clearly $R(f)=d\left(z, z^{\prime}\right) / 2$. Conjugating $\Gamma$ with an appropriate element of $G$ and relabelling we can arrange that $z=t+\mathbf{i}$ and $z^{\prime}=-t+\mathbf{i}$ are symmetric with respect to the $y$-axis in $\mathbb{H}$. Using the formula for hyperbolic distance (see e.g. [Ka, Theorem 1.2.6]) we find $\cosh d\left(z, z^{\prime}\right)=1+2 t^{2}$.

On the other hand $f=h_{t} r_{\theta} h_{-t}$ and $f^{\prime}=h_{-t} r_{\theta} h_{t}$. Computing $h=f^{\prime} f^{-1}$ directly we find $\operatorname{tr}(h)=2+4 t^{2} \sin ^{2} \theta$. Since this number is greater than $2, h$ is hyperbolic, and since this number is no more than $2 \cosh d\left(z, z^{\prime}\right)=2 \cosh 2 R(f)$, and the trace determines the eigenvalue, the second assertion follows.

Since $f^{-1}$ fixes $z$ and $f^{\prime}$ rotates around $z^{\prime}$, the distance which $z$ is moved by $h=f^{\prime} f^{-1}$ is bounded in terms of $R(f)$. On the other hand the order of $f$ leads to a lower bound on $\sin \theta$ and and hence to a lower bound on the displacement of $h$. Now let $p_{1}, p_{2}$ be the points on the axis of $h$ closest to $z$ and $z h$ respectively, so that $p_{2}=p_{1} h$; consider the quadrilateral joining $z, p_{1}, p_{2}, z h$. We have bounded $d\left(p_{1}, p_{2}\right)$ from below and $d(z, z h)$ from above, and the angles at $p_{1}, p_{2}$ are right angles. From this it is easy to deduce an upper bound on the length $d\left(p_{1}, z\right)$.

\section{Finiteness of small cusps}

In this section we prove a more precise version of Theorem 1.3. The idea is that when $\Gamma_{M}$ is non-elementary, a cusp in $\mathbb{H} / \Gamma_{M}$ gives rise to two transverse cylinder decompositions on $M$, one an image of the other under an affine automorphism, and the cusp area bounds the combinatorics of the corresponding intersection pattern. We will first consider translation surfaces, so denote

$$
\mathrm{SC}_{1}(m, T)=\{(M, P) \in \mathrm{SC}(m, T): M \text { is a translation surface }\},
$$

and by $\widetilde{\mathrm{SC}}_{1}(m, T)$ the corresponding sets of $G$-orbits.

For any $T>0$ and $m \in \mathbb{N}$, let $\mathcal{N}(m, T)$ denote the set of pairs $(A, D)$, where $A, D \in \operatorname{Mat}_{m}\left(\mathbb{Z}_{+}\right), A$ is symmetric, $D$ is diagonal, $D A$ is non-negative irreducible and $\lambda(D A)<T$. The following is an immediate corollary of Proposition 2.4.

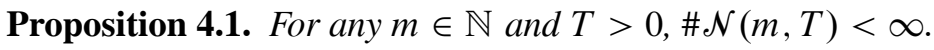

Given a symmetric $A=\left(a_{i j}\right) \in \operatorname{Mat}_{m}\left(\mathbb{Z}_{+}\right)$, let $\ell=\sum_{i, j} a_{i j}$. Denote by $\mathcal{P}(A)$ the set of simultaneous conjugacy classes $\left[\left(\sigma_{1}, \sigma_{2}\right)\right]$ for which (i) and (ii) of $\S 2.2$ hold, and such that $\sigma_{1}$ and $\sigma_{2}$ are conjugate in $S_{\ell}$. 
Theorem 4.2. For fixed $T>0$ and $m \in \mathbb{N}$,

$$
\# \widetilde{\mathrm{SC}}_{1}(m, T) \leq \sum_{(A, D) \in \mathcal{N}\left(m, \kappa_{m} T\right)} \# \mathcal{P}(A) .
$$

Proof. We will construct a map

$$
\Psi: \mathrm{SC}_{1}(m, T) \rightarrow X,
$$

where

$$
X=\left\{\left(A, D,\left[\left(\sigma_{1}, \sigma_{2}\right)\right]\right):(A, D) \in \mathcal{N}\left(m, \kappa_{m} T\right),\left[\left(\sigma_{1}, \sigma_{2}\right)\right] \in \mathcal{P}(A)\right\},
$$

such that $\Psi(M, P)=\Psi\left(M^{\prime}, P^{\prime}\right)$ if and only if there is $g \in G$ such that $M^{\prime}=g M$ and $P^{\prime}=g P g^{-1}$; in other words, $\Psi$ induces an injective map defined on $\widetilde{\mathrm{SC}}_{1}(m, T)$.

Let $\left(M^{\prime}, P^{\prime}\right) \in \mathrm{SC}_{1}(m, T)$, that is $M^{\prime}$ is a flat surface, $\Gamma_{M^{\prime}}$ is non-elementary, and $P^{\prime} \subset \Gamma_{M^{\prime}}$ is maximal parabolic. By a conjugation we may assume that $P^{\prime}$ is the cyclic group generated by $h_{1}$. Let $s, \gamma_{0}, h_{1}, t_{0}$ be as in Proposition 3.1, let $a$ be as (6), let $g=a^{-1} h_{-s}$, and let $M=g M^{\prime}$. Then $\Gamma_{M}=g \Gamma_{M^{\prime}} g^{-1}$ contains the two elements

$$
\gamma_{1}=h_{t_{0}}, \quad \gamma_{2}=\tilde{h}_{-t_{0}}
$$

and there is $\gamma \in \Gamma_{M}$ such that

$$
\gamma_{2}=\gamma^{-1} \gamma_{1} \gamma
$$

Moreover $P=g P^{\prime} g^{-1}$ is generated by $\gamma_{1}$.

By Proposition 2.1, there is a parabolic affine automorphism $\varphi_{1} \in \operatorname{Aff}(M)$ and $k \leq \kappa_{m}$ such that $D \varphi_{1}=\gamma_{1}^{k}=h_{k t_{0}}$, and $\varphi_{1}$ preserves the cylinders in a horizontal cylinder decomposition $M=C_{1} \cup \cdots \cup C_{m}$. Let $\psi \in \operatorname{Aff}(M)$ such that $\gamma=D \psi$, and let $\varphi_{2}=\psi^{-1} \varphi_{1} \psi \in \operatorname{Aff}(M)$, so that $D \varphi_{2}=\gamma_{2}^{k}$. Let $C_{i}^{\prime}=\psi^{-1}\left(C_{i}\right)$. Then $M=C_{1}^{\prime} \cup \cdots \cup C_{m}^{\prime}$ is a vertical cylinder decomposition invariant under $\varphi_{2}$.

Let $\vec{a}, \vec{w}$ (resp. $\vec{a}^{\prime}, \vec{w}^{\prime}$ ) be positive vectors in $\mathbb{R}^{m}$ recording the heights and circumferences of the cylinders $C_{1}, \ldots, C_{m}$ (resp. $C_{1}^{\prime}, \ldots, C_{m}^{\prime}$ ). It follows from (8) and (9) that $\gamma=h_{s_{1}} w \tilde{h}_{s_{2}}$ for some $s_{1}, s_{2} \in \mathbb{R}$, where $w$ is as in (6). Since the action of $h_{s}$ (resp. $\tilde{h}_{s}$ ) does not affect the heights and circumferences of horizontal (resp. vertical) cylinders, and since $w$ affects a rotation by $\pi / 2$, we get

$$
\vec{a}=\vec{a}^{\prime}, \quad \vec{w}=\vec{w}^{\prime} .
$$

Now let $A=\left(a_{i j}\right) \in \operatorname{Mat}_{m}(\mathbb{Z})$ where $a_{i j}$ is the number of connected components of $C_{i} \cap C_{j}^{\prime}$. By connectedness of $M, A$ is irreducible. Since $\psi$ exchanges the roles of $C_{i}$ and $C_{j}^{\prime}, A$ is symmetric. By construction, the gluing pattern $\left[\left(\sigma_{1}, \sigma_{2}\right)\right]$ corresponding to the two cylinder decompositions above is in $\mathcal{P}(A)$. We have

$$
\vec{w}=\vec{w}^{\prime}=A \vec{a} .
$$


Now let $n_{i}$ be the number of Dehn twists induced by $\varphi_{1}$ around a waist curve in $C_{i}$. By Proposition 2.1 we have $k t_{0}=n_{i} w_{i} / a_{i}$, that is, if $D=\operatorname{diag}\left(n_{1}, \ldots, n_{m}\right) \in$ $\operatorname{Mat}_{m}(\mathbb{N})$, then

$$
k t_{0} \vec{a}=D \vec{w}=D A \vec{a} .
$$

That is, $k t_{0}=\lambda(D A),(D, A) \in \mathcal{N}(k T)$ and $k \leq \kappa_{m}$. Altogether we have $\left(D, A,\left[\left(\sigma_{1}, \sigma_{2}\right)\right]\right)=\Psi\left(M^{\prime}, P\right) \in X$, so to prove the theorem it remains to show that the $G$-orbit of $\left(M^{\prime}, P^{\prime}\right)$ may be reconstructed from $\left(D, A,\left[\left(\sigma_{1}, \sigma_{2}\right)\right]\right)$. Note that $\vec{a}$ is uniquely determined up to scaling as the positive eigenvector of $D A$, and $\vec{w}$ is determined from $\vec{a}$ and $A$ via (10). The scaling parameter is also uniquely determined by the requirement that $M$ has area one. So $(A, D)$ determine $\vec{a}$ and $\vec{w}$, that is, the heights and circumferences of the rectangles, and $\left[\left(\sigma_{1}, \sigma_{2}\right)\right]$ determines how they are to be glued to each other. This determines $M$, and $P$ is the maximal parabolic subgroup of $\Gamma_{M}$ leaving the horizontal direction fixed.

Proof of Theorem 1.3. By Proposition 2.2, for each $(M, P) \in \mathrm{SC}(m, T)$, the orientation double cover $\widetilde{M}$ also has $P$ as a parabolic subgroup of $\Gamma_{\tilde{M}}$, and the corresponding cylinder decomposition has at most $2 m$ cylinders. Thus, if $\widetilde{P} \subset \Gamma_{\tilde{M}}$ is a maximal parabolic subgroup containing $P$, then $(M, P)$ gives rise to $(\tilde{M}, \widetilde{P}) \in \operatorname{SC}_{1}(2 m, T)$. Moreover for a fixed $(\widetilde{M}, \widetilde{P})$ there are only finitely many $(M, P)$ from which it arises in this way. The theorem follows.

\section{Hyperbolic affine automorphisms and Markov partitions}

In this section we prove Proposition 1.5. As noted in the introduction, the result is not new and presumably our argument is also well known. We include it since it does not appear in the literature.

Let $M$ be a flat surface and let $\varphi$ be a hyperbolic affine automorphism (in a different terminology, $\varphi$ represents a pseudo-Anosov homeomorphism). Let $h=D \varphi$ be the corresponding element of $\Gamma_{M}$, and let $\lambda=\lambda(h)$. By applying $g \in G$, let us assume that $h$ expands the $x$-axis and contracts the $y$-axis by a factor of $\lambda$. A Markov partition for $\varphi$ is a covering $M=P_{1} \cup \cdots \cup P_{p}$ by closed rectangles, with disjoint interiors, horizontal and vertical sides, such that the following Markov property is satisfied: for each $i$, the image of any vertical (resp. horizontal) side of any $P_{i}$ under $\varphi$ (resp. $\varphi^{-1}$ ) is contained in a vertical (resp. horizontal) side of one of the $P_{j}$. This means that if int $\varphi\left(P_{i}\right)$ intersects the interior of some $P_{j}$ then it extends all the way through to both sides. It is known that a Markov partition exists. A sketch of proof is given in [FLP], and we have provided more details in the appendix.

The intersection matrix of $\varphi$ is the $p \times p$ integer matrix $A=\left(a_{i j}\right)$, where $a_{i j}$ is the number of connected components of int $\varphi\left(P_{i}\right) \cap$ int $P_{j}$. One has: 
Proposition 5.1. $\bullet A$ is irreducible.

$\bullet \lambda=\lambda(A)$.

- The vector recording the widths (resp. heights) of the rectangle $P_{i}$ is a positive eigenvector for $A$ (resp. $A^{\top}$ ).

Proof. It follows from the Markov property that the $i, j$ th entry of $A^{n}$ counts the number of components in the intersection $\varphi^{n}\left(P_{i}\right) \cap P_{j}$. Suppose by contradiction that $A$ is not irreducible, so that there is a sequence $n_{k} \rightarrow \infty$ and $1 \leq i, j \leq p$ so that

$$
\varphi^{n_{k}}\left(P_{i}\right) \cap P_{j}=\varnothing .
$$

Let $\sigma$ be a horizontal segment in $P_{i}$ and consider its image $\sigma_{k}$ under $\varphi^{n_{k}}$. Then $\sigma_{k}$ is a horizontal segment contained in $\varphi^{n_{k}}\left(P_{i}\right)$ whose length tends to infinity. Passing to a subsequence we can assume the left endpoints of $\sigma_{k}$ converge to $x \in M$, so that any point along an infinite horizontal ray $\ell$ issuing from $x$ is a limit of points of $\sigma_{k}$. Since $\varphi^{-1}$ is an affine automorphism on $M$ which contracts horizontal saddle segments, there are no horizontal saddle connections on $M$, so the horizontal line flow on $M$ is minimal. This implies that $\ell$ is dense in $M$. In particular for large enough $k, \sigma_{k} \cap$ int $P_{j} \neq \varnothing$, contradicting (11).

Now let $w_{i}$ be the width of $P_{i}$, so that $\lambda w_{i}$ is the width of $\varphi\left(P_{i}\right)$. By the Markov property $\varphi\left(P_{i}\right)$ passes $a_{i j}$ times through $P_{j}$, so that $\lambda w_{i}=\sum_{j} a_{i j} w_{j}$. That is,

$$
\lambda \vec{w}=A \vec{w} .
$$

Since $\vec{w}$ is a positive vector, $\lambda=\lambda(A)$. The proof for heights is almost identical.

For a non-negative irreducible $A \in \operatorname{Mat}_{p}(\mathbb{Z})$, let $\mathcal{E}(A)$ denote the gluing patterns (as in \$2.2.2) of Markov partitions arising from hyperbolic affine automorphisms whose intersection matrix is $A$. We have:

Proposition 5.2. The set $\mathcal{E}(A)$ is finite.

Proof. Since $\varphi$ (resp. $\varphi^{-1}$ ) preserves the boundaries of rectangles, but contracts vertical (horizontal) saddle connections, there are there are no vertical or horizontal saddle connections for $M$. Thus each rectangle contributes at most $4 \pi$ to the total angle around the singularities of $M$. In other words the number of rectangles bounds the number of singularities. Since each edge in a gluing pattern is either bounded by a singularity or by a full size of a rectangle, this also bounds the number of edges in a gluing pattern. Thus the number of gluing patterns is finite.

We now want to show that intersection matrix of $\varphi$ determines the metric data for the gluing pattern, up to rescaling the surface. We normalize by assuming our surface 
has area one. Let $\xi_{i}$ be the horizontal segments of the gluing pattern corresponding to $P_{1}, \ldots, P_{p}$. We will refine our Markov partition to obtain another partition with the Markov property. Each $\xi_{i}$ is a connected component of the intersection of two of the $P_{j}$, say $P_{1}, P_{2}$. Consider the vertical segments (if any) which issue from the endpoints of $\xi_{i}$ into the interior of $P_{1}, P_{2}$. Any such segment divides a rectangle vertically into two rectangles, with the same height as before. At least one of the new rectangles will have width $\xi_{i}$.

We denote the resulting rectangle decomposition by $M=Q_{1} \cup \cdots \cup Q_{r}$, and claim that this decomposition also has the Markov property. To see this, note first that subdividing rectangles by vertical lines does not affect the requirement for $\varphi^{-1}$, which involves only horizontal sides. Now suppose $Q \subset P_{1}, P_{2}$ are as in Figure 3, $Q^{\prime} \subset P^{\prime}$ such that int $\varphi(Q) \cap$ int $Q^{\prime} \neq \varnothing$. There are two cases to consider:

(1) If $\varphi\left(P_{1}\right)$ does not reach the top of $P^{\prime}$ then also int $\varphi\left(P_{2}\right) \cap$ int $P^{\prime} \neq \varnothing$ and by the Markov property for the $P_{i}$, both $\varphi\left(P_{1}\right)$ and $\varphi\left(P_{2}\right)$ go across $P^{\prime}$ so $\varphi(Q)$ goes across $P^{\prime}$ and hence across $Q^{\prime}$.

(2) If $\varphi\left(P_{1}\right)$ reaches the top of $P^{\prime}$ and $P^{\prime \prime}$ lies above $P^{\prime}$ then $\varphi\left(P_{1}\right)$ goes across $P^{\prime}$ and $\varphi\left(P_{2}\right)$ goes across $P^{\prime \prime}$ so that $\varphi(Q)$ goes across the smaller of the two, i.e., $\varphi(Q)$ goes across $Q^{\prime}$.

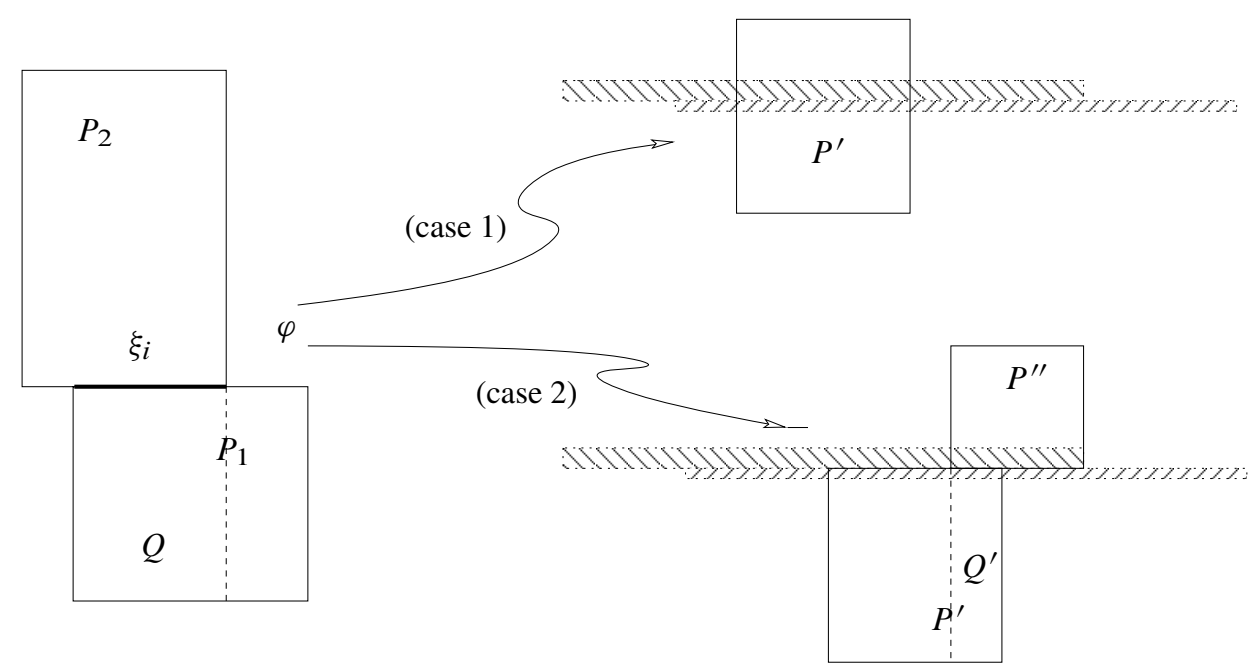

Figure 3. Markov property for the $Q_{j}$.

Considering all cases in this manner proves the claim.

We can also refine using the $\eta_{i}$ instead of the $\xi_{i}$, cutting rectangles horizontally, to obtain another Markov partition $T_{1}, \ldots, T_{t}$. We define matrices $B$ and $C$ by letting 
$b_{i j}$ (resp. $c_{i j}$ ) be the number of connected components of int $\varphi\left(Q_{i}\right) \cap$ int $Q_{j}$ (resp. int $\varphi\left(T_{i}\right) \cap$ int $\left.T_{j}\right)$. Repeating the proof of Proposition 5.1 we obtain:

Proposition 5.3. $\bullet B$ and $C$ are irreducible.

- $\lambda=\lambda(B)=\lambda(C)$.

- The vector recording the lengths of the segments $\xi_{i}$ (resp. $\eta_{i}$ ) is a positive eigenvector for B (resp. C).

We are now in a position to formulate and prove a more precise version of Proposition 1.5. We will consider separately translation and half-translation surfaces, so we write

$$
\operatorname{SMP}_{1}(p, T)=\{(M, h) \in \operatorname{SMP}(p, T): M \text { is a translation surface }\},
$$

and denote the corresponding set of $G$-orbits by $\widetilde{\operatorname{SMP}_{1}}(p, T)$. Let $\mathcal{M}(p, T)$ be the set of positive integer matrices $A$ with $\lambda(A)<T$. This is a finite set by Proposition 2.4. Then we have:

Theorem 5.4. For a fixed $T>0$ and $p \in \mathbb{N}$,

$$
\# \widetilde{\operatorname{SMP}}_{1}(p, T) \leq \sum_{A \in \mathcal{M}(p, T)} \# \mathscr{E}(A) .
$$

Proof. Given $(M, h) \in \operatorname{SMP}_{1}(p, T)$, we have constructed $A \in \mathcal{M}(p, T)$ and a gluing pattern in $\mathscr{E}(A)$, and these data only depend on the affine equivalence class of $M$. Thus we have defined a map

$$
\Phi: \widetilde{\operatorname{SMP}}_{1}(p, T) \rightarrow \bigcup_{A \in \mathcal{M}(p, T)} \mathcal{E}(A),
$$

and it remains to show that $\Phi$ is injective, i.e. that $M$ and $h$ are uniquely determined by the matrix $A$ and the gluing pattern, up to an element of $G$ and rescaling the surface. Normalizing we assume $M$ has area 1. Applying an element $g \in G$ we can assume that the parallelograms in the Markov partition corresponding to $(M, h)$ are rectangles with the expanding direction horizontal, and moreover the width of the widest rectangle can be normalized to be 1 . With this choice of $g$ we need to show that the matrix and gluing pattern uniquely determine $g M$. Since the widths of the rectangles are in the unique positive eigendirection of $A$, and by our scaling convention, the matrix $A$ determines the widths of the rectangles. The heights also span the unique eigendirection for $A^{\top}$ so are determined by $A$ up to scaling. By the requirement that $g M$ has area 1, the heights are uniquely determined by $A$. From $A$ and the gluing pattern one determines the matrices $B$ and $C$, and the lengths of the $\xi_{i}$ 
and $\eta_{j}$ give a positive eigenvector of $B$ and $C$ which is thus determined up to scaling. Since the sidelengths of the rectangles $P_{i}$ are determined, and can also be calculated using the lengths of the $\xi_{i}$ and $\eta_{i}$, the $\xi_{i}$ and $\eta_{j}$ are also uniquely determined. Since we have specified its gluing pattern and geometry, the flat surface $g M$ is uniquely determined.

Proof of Proposition 1.5. By Proposition 2.2, for each $(M, h) \in \operatorname{SMP}(p, T)$ the orientation double cover $\tilde{M}$ has a hyperbolic affine automorphism $\varphi$ such that the corresponding number of parallelograms is at most $2 p$. I.e., $(M, h) \in \operatorname{SMP}(p, T)$ gives rise to $(\tilde{M}, h) \in \operatorname{SMP}_{1}(2 p, T)$. Also for a fixed $(\tilde{M}, h)$ the number of $(M, h)$ covered in this way is finite. The proposition follows.

\section{Restrictions on Veech groups}

Proof of Corollary 1.6. It is easily checked that (I) and (II) depend only on the commensurability class of $\Gamma$, so to prove them we can assume that $\Gamma=\Gamma_{M}$ for a flat surface $M$. Since the maximal number of cylinders for a cylinder decomposition on $M$ is bounded, assertion (I) follows immediately from Theorem 1.3. Similarly assertion (II) is immediate from Proposition 1.5.

To prove assertion (III) we note that in a Veech group there is a uniform upper bound on the order of an elliptic element by Hurwitz's theorem, hence such a bound also exists in any group commensurable with a Veech group. So it remains to show that a Fuchsian group $\Gamma$ in which (II) holds and orders of elliptic elements are uniformly bounded, (III) holds. To see this, given $T$ suppose we have a list $z_{1}, z_{2}, \ldots$ of fixed points for elliptics $f_{1}, f_{2}, \ldots$ in $\Gamma$, such that the corresponding cone areas are no more than $T$. Since the order of the $f_{i}$ is bounded above, we have a uniform upper bound for $R\left(f_{i}\right)$ defined via (1). Let $h_{i}$ be the hyperbolic element corresponding to $f_{i}$ via Proposition 3.2. Since the eigenvalue of the $h_{i}$ is bounded, by (II) the list $h_{1}, \ldots$, contains only finitely many conjugacy classes. Conjugating the $f_{i}$ 's and correspondingly the $h_{i}$ 's we may assume that there are only finitely many distinct elements in the list $h_{1}, h_{2}, \ldots$ Given $h=h_{i}$ in this list, let $A$ be its axis, and let $A_{0} \subset A$ be a compact fundamental domain for the action of $h$ on $A$. By a further conjugation of $f_{i}$ assume that the closest point to $z_{i}$ on $A$ is in $A_{0}$. In view of the upper bound on the order of the $f_{i}$ 's, by Proposition $3.2 z_{i}$ is within bounded distance of $A_{0}$. By discreteness of $\Gamma$ there are only finitely many $z_{i}$ 's and hence finitely many $f_{i}$ 's.

Remark 6.1. The argument proving (III) can be adapted to prove the following analogue of Theorem 1.3 and Proposition 1.5: for any stratum $\mathscr{H}$ and any $T$, the set of affine equivalence classes of pairs $(M, f)$, where $M \in \mathscr{H}$ and $f \in \Gamma_{M}$ is elliptic, with corresponding cone area at most $T$, is finite. 
For the proof of Theorem 1.2 we will need the following lemma.

Lemma 6.2. Suppose $\Lambda_{0}$ is a Fuchsian group, $\Gamma$ a normal subgroup of $\Lambda_{0}, \gamma \in \Gamma$ a non-central element such that for any $\lambda \in \Lambda_{0}$ there is $\tau \in \Gamma$ with $\gamma^{\lambda}=\gamma^{\tau}$. Then $\Gamma$ is of finite index in $\Lambda_{0}$.

Proof of Theorem 1.2 (assuming Lemma 6.2). Let $\Gamma$ be a non-elementary Fuchsian group commensurable to a Veech group, let $\Lambda$ be the normalizer of $\Gamma$ in $G$, and suppose $\Lambda / \Gamma$ is infinite. Since $\Gamma$ is non-elementary it contains a hyperbolic element $\gamma$ [Ka, Theorem 2.4.4], and $\Lambda$ is Fuchsian [Ka, Theorem 2.3.8]. Let

$$
\Lambda_{0}=\left\{\lambda \in \Lambda: \gamma^{\lambda}=\gamma^{\tau} \text { for some } \tau \in \Gamma\right\} \text {. }
$$

In the action of $\Lambda$ on conjugacy classes of $\Gamma, \Lambda_{0}$ is the stabilizer of the conjugacy class of $\gamma$. In particular $\Lambda_{0}$ is a subgroup of $\Lambda$. According to Lemma $6.2, \Lambda_{0} / \Gamma$ is finite so $\Lambda / \Lambda_{0}$ is infinite. This implies that the conjugates $\left\{\gamma^{\lambda}: \lambda \in \Lambda\right\}$ comprise infinitely many conjugacy classes, in other words $\Gamma$ contains infinitely many hyperbolic elements which are conjugate in $\Lambda$, hence have the same eigenvalue, but are not conjugate in $\Gamma$. This contradicts Corollary 1.6.

Proof of Lemma 6.2. Let $C$ be the centralizer of $\gamma$ in $\Lambda_{0}$ and let $C_{0}=C \cap \Gamma$. Since $\Lambda_{0}$ is Fuchsian, $C$ is cyclic (see [Ka, §2.3]) and therefore $C_{0}$ is of finite index in $C$. By assumption for every $\lambda \in \Lambda_{0}$ there is $\tau \in \Gamma$ such that $\lambda^{-1} \tau \in C$, which implies that $\Lambda_{0}=C \Gamma$. Thus $C$ maps onto $\Lambda_{0} / \Gamma$. Since this surjection factors through $C / C_{0}, \Lambda_{0} / \Gamma$ must be finite.

Proof of Corollary 1.7. Given $\mathscr{H}$, for any $M \in \mathscr{H}$ and any hyperbolic $h \in \Gamma_{M}$, the minimal number $p(M, h)$ of parallelograms in a corresponding Markov partition can assume only finitely many values by Proposition A.1. Let $\Gamma$ contain a hyperbolic element $h$. By Proposition 1.5 the number of $G$-orbits of $M$ such that $h \in \Gamma_{M}$ is finite. In particular the set in (2) intersects only finitely many $G$-orbits. If $M_{1}$ and $M_{2}$ are flat surfaces in $\mathscr{H}$ such that $g M_{1}=M_{2}$ and $\Gamma_{M_{1}}=\Gamma_{M_{2}}$ then $g \in N$; and if $\Gamma$ is non-elementary then $N / \Gamma$ is finite by Corollary 1.2 .

Proof of Theorem 1.1. Given $R$ let $T=2 R$. Enlarging $T$ we ensure that a cusp of area at least $T$ contains an embedded ball of radius $R$. Enlarging $T$ further, in light of the upper bound on the order of elliptic elements in $\Gamma$, we ensure that any cone of area at least $T$ contains an embedded ball of radius $R$. Thus we may assume $\mathbb{H} / \Gamma$ contain neither a cusp of volume at least $T$, nor a cone of area at least $T$; in view of Corollary $1.6, \mathbb{H} / \Gamma$ has only finitely many cusps and cones, and only finitely many closed geodesics of length less than $T$.

Now let $\mathcal{N}_{1} \subset \mathbb{H} / \Gamma$ be the union of closed geodesics of length less than $T$, cusps of area less than $T$, and cones of area less than $T$. Then $\mathcal{N}_{1}$ has finite area. Let $\mathcal{N}$ 
be a neighborhood of $\mathcal{N}_{1}$ which is large enough so that a closed loop intersecting the complement of $\mathcal{N}$ and homotopic to either a geodesic of length less than $T$, a loop around a cusp of area less than $T$, or a loop around a cone point of area less than $T$, must have length at least $2 R$. Since $\mathbb{H} / \Gamma$ has only finitely many cusps and cones, the area of $\mathcal{N}$ is also finite, so $\mathbb{H} / \Gamma \neq \mathcal{N}$.

Let $\pi: \mathbb{H} \rightarrow \mathbb{H} / \Gamma$ be the natural map, and let $\bar{z} \in \mathbb{H}$ so that $z=\pi(\bar{z}) \notin \mathcal{N}$. We claim that $\left.\pi\right|_{B(\bar{z}, R)}$ is injective. Otherwise there is a segment $\bar{\sigma}$ connecting two distinct points in $B(\bar{z}, R)$, mapping to a closed loop $\sigma$ in $\mathbb{H} / \Gamma$ of length less than $2 R$. Either $\sigma$ has a shortest representative which is a geodesic of length less than $2 R$, or $\sigma$ is freely homotopic to a curve around a cusp or cone, that is a curve in $\mathcal{N}_{1}$. But then the definition of $\mathcal{N}$ ensures that the length of $\sigma$ is greater than $T$, and this is a contradiction.

Proof of Corollary 1.4. In a given stratum there is an upper bound on the number of cylinders in a cylinder decomposition. See [KeMaSm, Lemma, p. 302] or Smillie's improved bound as presented in [Na]. Suppose by contradiction that $M_{1}, M_{2}, \ldots$ are infinitely many affinely inequivalent surfaces, such that for each $i, \mathbb{H} / \Gamma_{i}$ does not contain an embedded ball of radius $R$. Here $\Gamma_{i}=\Gamma_{M_{i}}$. By Theorem 1.1 the $M_{i}$ are lattice surfaces, hence for each $i, \Gamma_{i}$ contains at least one maximal parabolic $P_{i}$. By Theorem $1.3, t_{0}\left(\Gamma_{i}, P_{i}\right) \rightarrow \infty$. A cusp with cusp area $t_{0}$ contains an embedded ball of radius $R\left(t_{0}\right)$, where $R\left(t_{0}\right) \rightarrow \infty$ as $t_{0} \rightarrow \infty-$ a contradiction proving (i). For a lattice surface, the cusp area is bounded above by the covolume, implying (ii).

Remark 6.3. It is also possible to deduce Corollary 1.4 and the finiteness of $\widetilde{\mathrm{SC}}(m, T) \cap \mathscr{H}$ for any stratum $\mathscr{H}$, from Proposition 1.5 , as follows. To any cusp one associates the element $h=\gamma_{1} \gamma_{2}$ as in (8) and proves it is hyperbolic, with $\lambda(h)$ bounded by a number depending on the corresponding cusp area. Our Theorem 1.3 is stronger in that it does not assume a bound on the topology of the surface, but only on the number of cylinders in the corresponding cylinder decomposition.

\section{Appendix. The construction of Markov partitions}

Proposition A.1. For any stratum $\mathscr{H}$ of flat surfaces, there are $R_{1}$ and $R_{2}$ such that for any surface $M \in \mathscr{H}$ and any hyperbolic $\varphi \in \operatorname{Aff}(M)$

- any Markov partition for $\varphi$ has at least $R_{1}$ parallelograms;

- there is a Markov partition for $\varphi$ with at most $R_{2}$ parallelograms.

Proof. Let $k=\sum_{\sigma \in \Sigma} k_{\sigma}$ so that $k \pi$ is the total angle around all the singularities of a surface in $\mathscr{H}$. Suppose $M \in \mathscr{H}$ and $\varphi \in \operatorname{Aff}(M)$ is hyperbolic, with a Markov partition into parallelograms $P_{1}, \ldots, P_{p}$. By conjugating we can assume the $P_{i}$ are 
rectangles with horizontal and vertical sides. The horizontal and vertical directions are contracted by either $\varphi$ or $\varphi^{-1}$, but these maps preserve the set of saddle connections, so (since there are only finitely many saddle connections in any direction) there are no horizontal or vertical saddle connections on $M$. In particular each edge of each $P_{i}$ can only contain one singularity, so that the total angle around singularities coming from each $P_{i}$ is at most $4 \pi$. This implies that $p \geq R_{1}=k / 4$, proving the first assertion.

To prove the second assertion, by Proposition 2.2 it suffices to consider strata of translation surfaces. Also, if we have a Markov partition for $\varphi^{r}$, the common refinement of its images under $\varphi^{i}, i=0, \ldots, r$, provides a Markov partition for $\varphi$. Thus by passing to a suitable finite power of $\varphi$, which can be taken to depend only on $\mathscr{H}$, we can assume that $\varphi$ fixes all the singularities and all the critical leaves issuing from singularities. Assume without loss of generality that the contracting direction for $\varphi\left(\right.$ resp. $\left.\varphi^{-1}\right)$ is vertical (resp. horizontal).

Since there are no horizontal or vertical saddle connections, the foliation in both the horizontal and vertical direction is minimal, so the leaves intersect any transverse segment. We will construct the edges of the partition in three steps.

(1) Let $\gamma$ be a short closed segment starting at a singularity and going along a horizontal leaf, say from left to right, and let $\lambda$ be a union of closed segments starting at each singularity along the vertical leaves (going both up and down) until the first point of intersection with $\gamma$. Denote the segments comprising $\lambda$ by $\lambda_{1}, \ldots, \lambda_{k}$.

(2) The intersection $\gamma \cap \lambda$ is finite, so there is a terminal point $p$ which is the rightmost intersection point of $\lambda \cap \gamma$. Remove from $\gamma$ the subsegment to the right of $p$. We retain the name $\gamma$ for the shorter segment.

(3) Suppose $\lambda_{j}$ ends at $p$. Continue it further until its next intersection with $\gamma$. We retain the names $\lambda, \lambda_{j}$ for this new collection. Since $M$ has no vertical saddle connections, the new intersection point of $\lambda_{j}$ with $\gamma$ is not one of the previous ones, and no two of these new intersection points coincide.

We first claim that each connected component of $M \backslash(\gamma \cup \lambda)$ is a rectangle. Take a segment $I \subset \gamma$ which is bounded by either a singularity and the first point where a $\lambda_{\ell}$ comes down to $\gamma$, or by two consecutive points at which $\lambda_{\ell}$ 's come down to $\gamma$. Consider the union of vertical leaves which begin at points of $I$ and move upwards. These form a strip, which near $\gamma$ is bounded on both sides by segments in $\lambda$. By choice of the $\lambda_{j}$ all these leaves all hit $\gamma$ again 'at the same time', i.e., none of these leaves hit a singularity or a terminal point before returning to $\gamma$. The construction also ensures that the segments from $\lambda$ on both sides of the strips also extend until the next intersection with $\gamma$. Thus the strip is a rectangle bounded by $\gamma \cup \lambda$.

We now claim that this partition into rectangles has the Markov property. Since $\varphi^{-1}$ fixes each singularity and maps each critical leaf to itself, and since it contracts 
the horizontal direction, $\gamma$ is mapped into itself. This implies that lower boundaries of rectangles map into lower boundaries under $\varphi^{-1}$. For identical reasons each $\lambda_{j}$ is mapped into itself by $\varphi$.

Now note that the endpoints of lower edges of rectangles on $\gamma$ are the terminal point $p$, the singularity, and one additional point for each downward pointing edge in $\lambda$. In total we have $2+k / 2$ endpoints which gives $1+k / 2$ rectangles. In particular the number of rectangles depends only on $\mathscr{H}$.

\section{References}

[DoHu] A. Douady and J. Hubbard, On the density of Strebel differentials. Invent. Math. 30 (1975), no. 2, 175-179. Zbl 0371.30017 MR 396936321

[EsOk] A. Eskin and A. Okounkov, Asymptotics of numbers of branched coverings of a torus and volumes of moduli spaces of holomorphic differentials. Invent. Math. 145 (2001), no. 1, 59-103. Zbl 1019.32014 MR 1839286318

[FLP] A. Fathi, F. Laudenbach and V. Poénaru (eds.), Travaux de Thurston sur les surfaces. Astérisque 66-67 (1979), Soc. Math. France, Paris 1979. Zbl 0406.00016 MR 0568308 315, 327

[Ga] F. R. Gantmacher, Theory of matrices. Chelsea Publishing, New York 1959. Zbl 0927.15002 MR 0107649 321, 322

[He] F. Herrlich, Teichmüller curves defined by characteristic origamis. Contemp. Math. 397 (2006), 133-144. Zbl 1098.14019 MR 2218004316

[HuSc1] P. Hubert and T. Schmidt, Invariants of translation surfaces. Ann. Inst. Fourier 51, 2 (2001), 461-495. Zbl 0985.32008 MR 1824961314

[HuSc2] P. Hubert and T. Schmidt, Infinitely generated Veech groups. Duke Math. J. 123 (2004), no. 1, 49-69. Zbl 1056.30044 MR 2060022313

[HuSc3] P. Hubert and T. Schmidt, An introduction to Veech surfaces. In Handbook of dynamical systems, Vol. 1B, ed. by B. Hasselblatt and A. Katok, Elsevier, Amsterdam 2006, 501-526. Zbl 1130.37367 MR 2186246314

[Ka] S. Katok, Fuchsian groups. Chicago Lectures in Mathematics, University of Chicago Press, Chicago, IL, 1992. Zbl 0753.30001 MR 1177168 325, 332

[KeMaSm] S. Kerckhoff, H. Masur and J. Smillie, Ergodicity of billiard flows and quadratic differentials. Ann. of Math. 124 (1986), 293-311. Zbl 0637.58010 MR 0855297 333

[MaSm] H. Masur and J. Smillie, Hausdorff dimension of sets of nonergodic measured foliations. Ann. of Math. 134 (1991), 455-543. Zbl 0774.58024 MR 1135877317

[MaTa] H. Masur and S. Tabachnikov, Rational billiards and flat structures. In Handbook of dynamical systems, Volume 1A, ed. by B. Hasselblatt and A. Katok, North-Holland Amsterdam 2002, 1015-1089. Zbl 1057.37034 MR 1928530 317

[Mc1] C. McMullen, Teichmüller geodesics of infinite complexity. Acta Math. 191 (2003), 191-223. Zbl 1131.37052 MR 2051398313 
[Mc2] C. McMullen, Personal communication, October 2004. 315

[Na] Y. Naveh, Tight upper bounds on the number of invariant components on translation surfaces. Israel J. Math. 165 (2008), 211-231. Zbl 1148.32008 MR 2403621333

[PaPe] A. Papadopoulos and R. C. Penner, Enumerating pseudo-Anosov foliations. Pacific J. Math. 142 (1990), 159-173. Zbl 0723.57021 MR 1038734322

[Pe] R. C. Penner, Bounds on least dilatations. Proc. Amer. Math. Soc. 113 (1991), 443-450. Zbl 0726.57013 MR 1068128315

[Ry] E. Rykken, Expanding factors for pseudo-Anosov homeomorphisms. Michigan Math. J. 46 (1999), no. 2, 281-296. Zbl 0987.37017 MR 704217 315, 321

[SmWe1] J. Smillie and B. Weiss, Finiteness results for flat surfaces: a survey and problem list. In Partially hyperbolic dynamics, laminations, and Teichmüller flow, ed. by G. Forni, M. Lyubich, and M. Shub, Fields Inst. Commun. 51, Amer. Math. Soc., Providence, RI, 2007, 125-137. Zbl 1151.57020 MR 2388694 314, 316

[SmWe2] J. Smillie and B. Weiss, Characterizations of lattice surfaces. Preprint 2008. arXiv:0809.3729

[Th] W. Thurston, On the geometry and dynamics of diffeomorphisms of surfaces. Bull. Amer. Math. Soc. (N.S.) 19 (1988), no. 2, 417-431. Zbl 0674.57008 MR 0956596 315

[Ve1] W. A. Veech, The Teichmüller geodesic flow. Ann. of Math. 124 (1986), 441-530. Zbl 0658.32016 MR 0866707316

[Ve2] W. A. Veech, Teichmüller curves in moduli space, Eisenstein series and an application to triangular billiards. Invent. Math. 97 (1989), no. 3, 553-583. Zbl 0676.32006 MR 1005006 313, 315, 320

[Vo] Ya. B. Vorobets, Planar structures and billiards in rational polygons: the Veech alternative, Uspekhi Mat. Nauk 51 (1996), no. 5, 3-42; Engl. transl. Russian Math. Surveys 51 (1996), no. 5, 779-817. Zbl 0897.58029 MR 1436653 317, 321

[Zo] A. Zorich, Flat surfaces. In Frontiers in number theory, physics and geometry I, ed. by P. Cartier, B. Julia, P. Moussa and P. Vanhove, Springer-Verlag, Berlin 2006, 437-583. Zbl 1129.32012 MR 2261104317

Received April 1, 2008

John Smillie, Department of Mathematics, 304 White Hall, Cornell University, Ithaca,

NY 14853-7901, U.S.A.

E-mail: smillie@math.cornell.edu

Barak Weiss, Department of Mathematics, Ben Gurion University of the Negev, Be'er Sheva,

84105, Israel

E-mail: barakw@math.bgu.ac.il 